doi: $10.2306 /$ scienceasia1513-1874.2011.37.136

\title{
Solutions of a class of nonlinear recursive equations and applications
}

\author{
Charinthip Hengkrawit $^{\mathrm{a}}$, Vichian Laohakosol ${ }^{\mathrm{b}, \mathrm{c}}$, Watcharapon Pimsert ${ }^{\mathrm{b}, *}$ \\ ${ }^{\text {a }}$ Department of Mathematics and Statistics, Faculty of Science and Technology, Thammasat University, \\ Pathum Thani 12120, Thailand \\ b Department of Mathematics, Institute for Advanced Studies, Kasetsart University, Bangkok 10900, Thailand \\ ${ }^{c}$ Centre of Excellence in Mathematics, CHE, Si Ayutthaya Road, Bangkok 10400, Thailand \\ *Corresponding author, e-mail: charinthip@mathstat.sci.tu.ac.th,fscivil@ku.ac.th, fsciwcrp@ku.ac.th
}

Received 29 Aug 2010

Accepted 12 Apr 2011

\begin{abstract}
A class of recursive equations extending those of the form $y_{n}=\left(y_{n-2} y_{n-1}-1\right) /\left(y_{n-2}+y_{n-1}\right)$ is transformed into a special case of the nonlinear recursive equation $x_{n}=c x_{n-1}^{h_{1}(n)} x_{n-2}^{h_{2}(n)} \cdots x_{n-\ell+1}^{h_{\ell-1}(n)} x_{n-\ell}^{h_{\ell}(n)}$. A general solution of this equation is determined by solving its associated linear difference equation. Several known results are derived as special cases. Connections of the case $\ell=2$ to continued fractions are elaborated.
\end{abstract}

KEYWORDS: closed form solutions, continued fractions

\section{INTRODUCTION}

Rational recursive equations of the form

$$
y_{n+\ell}=f\left(y_{n+\ell-1}, \ldots, y_{n+1}, y_{n}\right),
$$

where $f$ is a given rational function, have been of much interest recently both in their own right and because of their applications to various other fields ${ }^{1,2}$. More related recent works can be found in Refs. 3-8. It is natural then to seek closed form solutions of such equations whenever possible. With explicit forms of solution, numerical computations can be directly implemented and further aspects such as asymptotic behaviour, periodicity, or other qualitative analysis can be treated in a straightforward manner. Rhouma ${ }^{9}$ gave a closed form solution to the rational recursive difference equation

$$
y_{n+2}=\frac{y_{n} y_{n+1}-1}{y_{n}+y_{n+1}},
$$

which originated from an open problem in the book ${ }^{1}$ (see also Ref. 2 where global asymptotic stability of its solution is discussed). Rhouma's technique is first to transform (1) to an equivalent form of

$$
y_{n+2}=\mathrm{i} \frac{\left(y_{n+1}+\mathrm{i}\right)\left(y_{n}+\mathrm{i}\right)+\left(y_{n+1}-\mathrm{i}\right)\left(y_{n}-\mathrm{i}\right)}{\left(y_{n+1}+\mathrm{i}\right)\left(y_{n}+\mathrm{i}\right)-\left(y_{n+1}-\mathrm{i}\right)\left(y_{n}-\mathrm{i}\right)},
$$

where $\mathrm{i}=\sqrt{-1}$, or

$$
\frac{y_{n+2}-\mathrm{i}}{y_{n+2}+\mathrm{i}}=\frac{y_{n+1}-\mathrm{i}}{y_{n+1}+\mathrm{i}} \cdot \frac{y_{n}-\mathrm{i}}{y_{n}+\mathrm{i}},
$$

which is a difference equation of the form

$$
x_{n+2}=\alpha x_{n+1} x_{n} .
$$

A closed form solution to (3) is then derived in terms of the Fibonacci numbers. In Ref. 10, this technique is employed to derive an explicit solution of the equation

$$
y_{n+\ell}=\mathrm{i}\left(\frac{T_{p}+T_{m}}{T_{p}-T_{m}}\right) \text {, }
$$

where

$$
T_{p}=\left(y_{n+\ell-1}+\mathrm{i}\right)^{A_{1}} \ldots\left(y_{n}+\mathrm{i}\right)^{A_{\ell}}
$$

and

$$
T_{m}=\left(y_{n+\ell-1}-\mathrm{i}\right)^{A_{1}} \ldots\left(y_{n}-\mathrm{i}\right)^{A_{\ell}},
$$

extending (2). In the last section of Ref. 9, Rhouma illustrates how rational recursive equations, generalizing (3), of the form

$$
x_{n+\ell}=c \prod_{j=0}^{\ell-1} x_{n+j}^{A_{\ell-j}} \quad(n \in \mathbb{N} \cup\{0\}),
$$

with initial conditions $x_{0}, x_{1}, \ldots, x_{\ell-1}$ have closed form solutions

$$
x_{n}=c^{\mathfrak{B}_{n}} \prod_{j=0}^{\ell-1} x_{j}^{\mathfrak{A}_{n}^{(j)}}
$$


where the sequences $\left\{\mathfrak{A}_{n}^{(j)}\right\}$ satisfy

$$
\begin{aligned}
\mathfrak{A}_{n+\ell}^{(j)} & =\sum_{m=0}^{\ell-1} A_{\ell-m} \mathfrak{A}_{n+m}^{(j)}, \\
\mathfrak{A}_{j}^{(j)} & =1, \mathfrak{A}_{m}^{(j)}=0 \quad(0 \leqslant j \neq m \leqslant \ell-1),
\end{aligned}
$$

and the sequence $\left\{\mathfrak{B}_{n}\right\}$ satisfies

$$
\mathfrak{B}_{n+\ell}=1+\sum_{m=0}^{\ell-1} A_{\ell-m} \mathfrak{B}_{n+m},
$$

where $\mathfrak{B}_{0}=\mathfrak{B}_{1}=\ldots=\mathfrak{B}_{\ell-1}=0$.

Our objective here is to present in detail an alternative and direct approach to solving the rational recursive equation

$$
x_{n}=c x_{n-1}^{h_{1}(n)} x_{n-2}^{h_{2}(n)} \cdots x_{n-\ell+1}^{h_{\ell-1}(n)} x_{n-\ell}^{h_{\ell}(n)},
$$

where $n \geqslant \ell$, generalizing (4).

\section{GENERAL EXPLICIT SOLUTION}

In this section, we solve the equation (7).

Theorem 1 Let $\ell \in \mathbb{N}$ and $c \in \mathbb{C} \backslash\{0\}$. Let $h_{1}, \ldots$, $h_{\ell}$ be functions from $\mathbb{N}_{0}:=\mathbb{N} \cup\{0\}$ to $\mathbb{C}$ with $h_{\ell}(n) \neq 0$ for all $n$. Let $\left\{G_{n}^{(1)}\right\}_{n \geqslant 0}, \ldots,\left\{G_{n}^{(\ell)}\right\}_{n \geqslant 0}$ be $\ell$ unique sequences satisfying the linear recurrence

$$
G_{n}^{(j)}-h_{1}(n) G_{n-1}^{(j)}-\cdots-h_{\ell}(n) G_{n-\ell}^{(j)}=0,
$$

for $n \geqslant \ell$ and $j=1,2, \ldots, \ell$ with given initial values

$$
G_{j-1}^{(i)}=\delta(i, j) \quad(i, j=1,2, \ldots \ell),
$$

where $\delta(i, j)$ is the usual Kronecker delta taking values 1 if $i=j$ and 0 otherwise. If $\left\{x_{n}\right\}_{n \geqslant 0}$ is a sequence of complex numbers satisfying the recursive equation

$$
x_{n}=c x_{n-1}^{h_{1}(n)} x_{n-2}^{h_{2}(n)} \cdots x_{n-\ell}^{h_{\ell}(n)} \quad(n \geqslant \ell),
$$

with given initial values $x_{0}, \ldots, x_{\ell-1}$ chosen so that all remaining $x_{n}$ are uniquely well-defined, then the solution of (10) is given by

$$
x_{n}=P_{c}(n) \prod_{i=0}^{\ell-1}\left(\frac{x_{i}}{P_{c}(i)}\right)^{G_{n}^{(i+1)}} \quad(n \geqslant 0),
$$

where $P_{c}(n)$ is a particular solution of (10).

Proof: Given the initial values $x_{0}, \ldots, x_{\ell-1}$, the equation (10) uniquely determines all the elements $x_{n}$ for $n \geqslant \ell$. It thus suffices to verify that the general form of the solution to (10) is given by (11). Putting $i=1, \ldots, \ell-1$, into the right-hand side of (11), we get, respectively,

$P_{c}(0)\left(\frac{x_{0}}{P_{c}(0)}\right)^{1}\left(\frac{x_{1}}{P_{c}(1)}\right)^{0} \cdots\left(\frac{x_{\ell-1}}{P_{c}(\ell-1)}\right)^{0}=x_{0}$,
$P_{c}(1)\left(\frac{x_{0}}{P_{c}(0)}\right)^{0}\left(\frac{x_{1}}{P_{c}(1)}\right)^{1} \cdots\left(\frac{x_{\ell-1}}{P_{c}(\ell-1)}\right)^{0}=x_{1}$,

$P_{c}(\ell-1)\left(\frac{x_{0}}{P_{c}(0)}\right)^{0} \cdots\left(\frac{x_{\ell-1}}{P_{c}(\ell-1)}\right)^{1}=x_{\ell-1}$.

This shows that (11) holds for all the initial values. Putting $n=\ell$ into the right-hand side of (11), we get

$$
\begin{aligned}
& P_{c}(\ell) \prod_{i=0}^{\ell-1}\left(\frac{x_{i}}{P_{c}(i)}\right)^{G_{n}^{(i+1)}} \\
& =P_{c}(\ell)\left(\frac{x_{0}}{P_{c}(0)}\right)^{G_{\ell}^{(1)}}\left(\frac{x_{1}}{P_{c}(1)}\right)^{G_{\ell}^{(2)}} \cdots\left(\frac{x_{\ell-1}}{P_{c}(\ell-1)}\right)^{G_{\ell}^{(\ell)}} \\
& =P_{c}(\ell) \frac{x_{0}^{h_{\ell}(\ell)} x_{1}^{h_{\ell}(\ell-1)} \cdots x_{\ell-1}^{h_{1}(\ell)}}{P_{c}(0)^{h_{\ell}(\ell)} P_{c}(1)^{h_{\ell}(\ell-1)} \cdots P_{c}(\ell-1)^{h_{1}(\ell)}} \\
& =c x_{0}^{h_{\ell}(\ell)} x_{1}^{h_{\ell}(\ell-1)} \cdots x_{\ell-1}^{h_{1}(\ell)}=x_{\ell}
\end{aligned}
$$

by (10). Next, suppose that (11) is true for all $n=$ $0,1, \ldots, k$. Then

$$
\begin{aligned}
x_{k+1}= & c \prod_{j=1}^{\ell-1} x_{(k+1)-j}^{h_{j}(k+1)} \\
= & c \prod_{j=1}^{\ell}\left(P_{c}(k+1-j) \prod_{i=0}^{\ell-1}\left(\frac{x_{i}}{P_{c}(i)}\right)^{G_{k+1-j}^{(i+1)}}\right)^{h_{j}(k+1)} \\
= & c\left(\prod_{j=1}^{\ell} P_{c}(k+1-j)^{h_{j}(k+1)}\right) \\
& \cdot\left(\prod_{i=0}^{\ell-1}\left(\frac{x_{i}}{P_{c}(i)}\right)^{\sum_{m=0}^{\ell} h_{m}(k+1) G_{k+1-m}^{(i+1)}}\right) \\
= & c P_{c}(k+1) \prod_{i=0}^{\ell-1}\left(\frac{x_{i}}{P_{c}(i)}\right)^{G_{k+1}^{(i+1)}} .
\end{aligned}
$$

By mathematical induction, we conclude that (11) holds for all $n \geqslant 0$.

Remark 1 The shape of the solution given in (11) may be easily obtained through the following formal manipulation. Taking the logarithm of (10), we get the 
linear difference equation

$$
\log x_{n}=\log c+\sum_{i=1}^{\ell} h_{i}(n) \log x_{n-i} .
$$

Putting $f(n):=\log x_{n}$, the recurrence (12) becomes

$$
f(n)=\log c+\sum_{i=1}^{\ell} h_{i}(n) f(n-i) .
$$

Since each sequence in the system

$$
f_{1}(n)=G_{n}^{(1)}, \ldots, f_{\ell}(n)=G_{n}^{(\ell)} \quad(n \geqslant 0),
$$

satisfies the homogeneous recurrence (8) and the system is $\mathbb{C}$-linearly independent because of the initial conditions (9), the general solution of (13) is given by ${ }^{11}$

$$
\begin{aligned}
f(n) & =\sum_{i=1}^{\ell} f_{i}(n) \log \beta_{i}+\log P_{c}(n) \\
& =\log \left(P_{c}(n) \beta_{1}^{G_{n}^{(1)}} \beta_{2}^{G_{n}^{(2)}} \cdots \beta_{\ell}^{G_{n}^{(\ell)}}\right),
\end{aligned}
$$

where $\beta_{i}(i=1,2, \ldots, \ell)$ are constants. Thus the general solution of (7) is given by

$$
x_{n}=e^{f(n)}=P_{c}(n) \beta_{1}^{G_{n}^{(1)}} \beta_{2}^{G_{n}^{(2)}} \cdots \beta_{\ell}^{G_{n}^{(\ell)}}(n \geqslant \ell) .
$$

To determine the $\beta_{j}$ 's, substituting $n=0,1, \ldots, \ell-1$ successively in this last expression and making use of the initial conditions (9), we get

$$
\beta_{1}=x_{0} / P_{c}(0), \ldots, \beta_{\ell}=x_{\ell-1} / P_{c}(\ell-1) .
$$

Remark 2 In the result of Theorem 1, if $c=1$, we can simply take $P_{c}(n)=1$ for all $n$. If $1-h_{1}(n)-$ $h_{2}(n)-\cdots-h_{\ell}(n)$ is a non-zero constant independent of $n$, say equal to $1 / H$, then we can take $P_{c}(n)=c^{H}$, a constant independent of $n$.

\section{CONSTANT EXPONENTS}

If the exponent functions $h_{1}(n), \ldots, h_{\ell}(n)$ in (7) are constants, then the result in Theorem 1 gives the following corollary.

Corollary 1 Let $\ell \in \mathbb{N}$ and $c(\neq 0), A_{1}, \ldots, A_{\ell}(\neq 0)$ $\in \mathbb{C}$. Let $\left\{x_{n}\right\}_{n \geqslant 0}$ be a sequence of complex numbers satisfying the recursive equation

$$
x_{n+\ell}=c x_{n+\ell-1}^{A_{1}} x_{n+\ell-2}^{A_{2}} \cdots x_{n+1}^{A_{\ell-1}} x_{n}^{A_{\ell}}
$$

with the initial values $x_{0}, \ldots, x_{\ell-1}$ being chosen so that all remaining $x_{n}$ are uniquely well-defined. Let $\left\{G_{n}\right\}$ be the unique sequence satisfying

$$
G_{n+\ell}-A_{1} G_{n+\ell-1}-\cdots-A_{\ell} G_{n}=0,
$$

with given initial values $G_{0}, \ldots, G_{\ell-1}$. Assume that the sequence $\left\{G_{n}\right\}$ does not satisfy any recurrence of the form (16) of lower order. Then the solution of (15) is given by

$$
x_{n}=P_{c}(n) \beta_{1}^{G_{n-1}} \beta_{2}^{G_{n-2}} \cdots \beta_{\ell}^{G_{n-\ell}} \quad(n \geqslant \ell),
$$

where $P_{c}(n)$ is a particular solution of (4) and the $\beta_{j}(j=1, \ldots, \ell)$ are successively determined from the system, for $k=0, \ldots, \ell-1$,

$$
c x_{k+\ell-1}^{A_{1}} \cdots x_{k+1}^{A_{\ell-1}} x_{k}^{A_{\ell}}=P_{c} \beta_{1}^{G_{\ell+k-1}} \cdots \beta_{\ell}^{G_{k}} .
$$

Moreover, if $\kappa_{\ell}:=1-\left(A_{1}+\cdots+A_{\ell}\right) \neq 0$, then the solution of (15) is given by

$$
x_{n}=c^{1 / \kappa_{\ell}} \beta_{1}^{G_{n-1}} \beta_{2}^{G_{n-2}} \cdots \beta_{\ell}^{G_{n-\ell}} \quad(n \geqslant \ell) .
$$

The result in Corollary 1 can be made more explicit in terms of the roots of the characteristic equation of (16) as we now see.

Corollary 2 Let the notation be as in Corollary 1. Let all the distinct roots of the characteristic polynomial,

$$
C(X)=X^{\ell}-A_{1} X^{\ell-1}-A_{2} X^{\ell-2}-\cdots-A_{\ell},
$$

be $R_{1}, \ldots, R_{r}$ with respective multiplicities $m_{1}, \ldots$, $m_{r}$ so that $m_{1}+\cdots+m_{r}=\ell$. Then the solution of (15) is

$$
x_{n}=P_{c}(n) \exp \left(\sum_{k=1}^{r}\left(\sum_{i=1}^{m_{k}} a_{k i} n^{i-1}\right) R_{k}^{n}\right),
$$

where the coefficients $a_{k s}\left(1 \leqslant s \leqslant m_{k}, 1 \leqslant k \leqslant r\right)$ are uniquely determined from the given initial values $x_{0}, \ldots, x_{\ell-1}$.

Proof: By a well-known theorem about linear difference equations with constant coefficients (see e.g., Chapter 2 of Ref. 12) the general solution of (16) is

$$
\begin{aligned}
f(n) & =\left(a_{11}+\cdots+a_{1, m_{1}} n^{m_{1}-1}\right) R_{1}^{n}+\cdots \\
& +\left(a_{r 1}+\cdots+a_{r, m_{r}} n^{m_{r}-1}\right) R_{r}^{n},
\end{aligned}
$$

which then yields the general solution of (15) as the one in (19).

As illustrations, we work out closed form solutions of the two simplest cases followed by that of the general case.

Corollary 3 Let the notation be as in Corollary 2. If all the roots of the characteristic polynomial $C(X)$ are simple, then the solution of (15) is

$$
x_{n}=P_{c}(n) \exp \left(b_{1} R_{1}^{n}+\cdots+b_{\ell} R_{\ell}^{n}\right),
$$


with the coefficients $b_{j}$ given by

$$
b_{j}=D_{j} / D \quad(j=1, \ldots, \ell),
$$

where

$$
\begin{aligned}
D: & =\left|\begin{array}{cccc}
1 & 1 & \cdots & 1 \\
R_{1} & R_{2} & \cdots & R_{\ell} \\
R_{1}^{2} & R_{2}^{2} & \cdots & R_{\ell}^{2} \\
\vdots & \vdots & & \vdots \\
R_{1}^{\ell-1} & R_{2}^{\ell-1} & \cdots & R_{\ell}^{\ell-1}
\end{array}\right| \\
& =\prod_{1 \leqslant s<t \leqslant \ell}\left(R_{s}-R_{t}\right)
\end{aligned}
$$

and $D_{j}$ denotes the determinant obtained from $D$ by replacing the $j$ th column by the vector $\left(\log \left(x_{0} / P_{c}(0)\right), \ldots, \log \left(x_{\ell-1} / P_{c}(\ell-1)\right)\right)^{\mathrm{T}}$ where Log denotes the principal branch of the complex logarithmic function.

Corollary 4 Let the notation be as in Corollary 2. If the characteristic polynomial $C(X)$ has only a single root $R$ with multiplicity $\ell$, then the solution of (4) is

$$
x_{n}=P_{c} \exp \left(R^{n}\left(d_{0}+d_{1} n+\cdots+d_{\ell-1} n^{\ell-1}\right)\right),
$$

with the coefficients $d_{j}$ given by

$$
d_{j}=D_{j} / D \quad(j=0, \ldots, \ell-1),
$$

where

$$
\begin{aligned}
D: & =\left|\begin{array}{cccc}
1 & 0 & \cdots & 0 \\
1 & 1 & \cdots & 1 \\
1 & 2 & \cdots & 2^{\ell-1} \\
\vdots & \vdots & \vdots & \vdots \\
1 & (\ell-1) & \cdots & (\ell-1)^{\ell-1}
\end{array}\right| \\
& =(-1)^{\left(\begin{array}{c}
\ell-1 \\
2
\end{array}\right)}(\ell-2) !(\ell-1) ! \cdots 2 ! 1 !
\end{aligned}
$$

and $D_{j}$ denotes the determinant obtained from $D$ by replacing the jth column by the vector

$$
\left(\begin{array}{c}
\log \left(x_{0} / P_{c}(0)\right) \\
R^{-1} \log \left(x_{1} / P_{c}(1)\right) \\
\vdots \\
R^{-\ell+1} \log \left(x_{\ell-1} / P_{c}(\ell-1)\right)
\end{array}\right) .
$$

Explicit forms of the coefficients $a_{k s}$ in the general closed form solution of Corollary 2 can be obtained from a much more complicated determinant formula (see e.g., p. 283 of Ref. 13).
Corollary 5 The solution of (4) is of the form (19) with the coefficients $a_{k s}$ being given by $a_{k s}=D_{k s} / D$ where

$$
\begin{aligned}
D: & =\left|(-1+\lambda)^{s} R_{k}^{-1+\lambda}\right|_{(k, s), \lambda} \\
& =\prod_{h=1}^{r} \prod_{t=1}^{m_{h}}\left((t-1) ! R_{h}^{-1+t} \prod_{k=1}^{h-1}\left(R_{h}-R_{k}\right)^{m_{k}}\right) .
\end{aligned}
$$

Here the notation is such as to display a typical term, rows indexed by the $\ell$ pairs $(k, s)$ arranged lexicographically and columns by $\lambda=$ $1,2, \ldots, \ell . \quad D_{k s}$ denotes the determinant obtained from $D$ by replacing the $(k, s)$ column by the vector $\left(\log \left(x_{0} / P_{c}(0)\right), \ldots, \log \left(x_{\ell-1} / P_{c}(\ell-1)\right)\right)^{\mathrm{T}}$.

The next three examples demonstrate that the closedform solution of (3) and those in Lemmas 3 and 4 of Ref. 9 are special cases of our results.

Example 1 Let $\ell=2, A_{1}=1, A_{2}=1$, and $c=\alpha$ so that $\left\{G_{n}\right\}$ is the sequence of Fibonacci numbers $\left\{F_{n}\right\}_{n \geqslant 0}:=\{1,1,2, \ldots\}$. By Corollary 1 , a closedform solution of (3) is

$$
x_{n}=\alpha^{-1} \beta_{1}^{F_{n-1}} \beta_{2}^{F_{n-2}} \quad(n \geqslant 2),
$$

where $\beta_{1}, \beta_{2}$ are successively determined from (18). We have

$$
\begin{aligned}
& \alpha x_{1} x_{0}=\alpha^{-1} \beta_{1}^{F_{1}} \beta_{2}^{F_{0}}=\alpha^{-1} \beta_{1} \beta_{2}, \\
& \alpha x_{2} x_{1}=\alpha^{-1} \beta_{1}^{F_{2}} \beta_{2}^{F_{1}}=\alpha^{-1} \beta_{1}^{2} \beta_{2},
\end{aligned}
$$

and so $\beta_{1}=\alpha x_{1}$ and $\beta_{2}=\alpha x_{0}$. Thus the explicit solution of (3) is

$$
\begin{aligned}
x_{n} & =\alpha^{-1+\left(F_{n-1}+F_{n-2}\right)} x_{1}^{F_{n-1}} x_{0}^{F_{n-2}} \\
& =\alpha^{-1+F_{n}} x_{1}^{F_{n-1}} x_{0}^{F_{n-2}} \quad(n \geqslant 2),
\end{aligned}
$$

which agrees with Lemma 2 in Ref. 9.

Example 2 Let $\ell=3, A_{1}=0, A_{2}=1, A_{3}=1$ and $c=1$. The recursive equation to solve is

$$
x_{n+3}=x_{n+1} x_{n} .
$$

The sequence $\left\{G_{n}\right\}$ satisfies

$$
\begin{aligned}
0 & =G_{n+3}-A_{1} G_{n+2}-A_{2} G_{n+1}-A_{3} G_{n} \\
& =G_{n+3}-G_{n+1}-G_{n}
\end{aligned}
$$

and for convenience we take as initial values

$$
G_{0}=0, G_{1}=1, G_{2}=0 .
$$


By Corollary 1, a closed-form solution of (20) is

$$
x_{n}=\beta_{1}^{G_{n-1}} \beta_{2}^{G_{n-2}} \beta_{3}^{G_{n-3}},
$$

where $\beta_{1}, \beta_{2}, \beta_{3}$ are determined from

$$
\begin{aligned}
& x_{1} x_{0}=x_{3}=\beta_{1}^{G_{2}} \beta_{2}^{G_{1}} \beta_{3}^{G_{0}}=\beta_{2} \\
& x_{2} x_{1}=x_{4}=\beta_{1}^{G_{3}} \beta_{2}^{G_{2}} \beta_{3}^{G_{1}}=\beta_{1} \beta_{3} \\
& x_{3} x_{2}=x_{5}=\beta_{1}^{G_{4}} \beta_{2}^{G_{3}} \beta_{3}^{G_{2}}=\beta_{1} \beta_{2} .
\end{aligned}
$$

Here, $\beta_{2}=x_{0} x_{1}, \beta_{1}=x_{2}$ and $\beta_{3}=x_{1}$ yielding

$$
\begin{aligned}
x_{n} & =\left(x_{2}\right)^{G_{n-1}}\left(x_{0} x_{1}\right)^{G_{n-2}}\left(x_{1}\right)^{G_{n-3}} \\
& =x_{0}^{G_{n-2}} x_{1}^{G_{n-2}+G_{n-3}} x_{2}^{G_{n-1}} \\
& =x_{0}^{G_{n-2}} x_{1}^{G_{n}} x_{2}^{G_{n-1}} .
\end{aligned}
$$

This agrees with Lemma 3 in Ref. 9.

Example 3 Let $\ell=3, A_{1}=1, A_{2}=0, A_{3}=1$, and $c=1$. The recursive equation to solve is

$$
x_{n+3}=x_{n+2} x_{n} .
$$

The sequence $\left\{G_{n}\right\}$ satisfies

$$
\begin{aligned}
0 & =G_{n+3}-A_{1} G_{n+2}-A_{2} G_{n+1}-A_{3} G_{n} \\
& =G_{n+3}-G_{n+2}-G_{n},
\end{aligned}
$$

and we take as initial values $G_{0}=0, G_{1}=0, G_{2}=$ 1. By Corollary 1, the solution of (21) is $x_{n}=$ $\beta_{1}^{G_{n-1}} \beta_{2}^{G_{n-2}} \beta_{3}^{G_{n-3}}$, where $\beta_{1}, \beta_{2}, \beta_{3}$ satisfy

$$
\begin{aligned}
& x_{2} x_{0}=x_{3}=\beta_{1}^{G_{2}} \beta_{2}^{G_{1}} \beta_{3}^{G_{0}}=\beta_{1} \\
& x_{3} x_{1}=x_{2} x_{1} x_{0}=x_{4}=\beta_{1}^{G_{3}} \beta_{2}^{G_{2}} \beta_{3}^{G_{1}}=\beta_{1} \beta_{2} \\
& x_{4} x_{2}=x_{2}^{2} x_{1} x_{0}=x_{5}=\beta_{1}^{G_{4}} \beta_{2}^{G_{3}} \beta_{3}^{G_{2}}=\beta_{1} \beta_{2} \beta_{3} .
\end{aligned}
$$

Here, $\beta_{1}=x_{2} x_{0}, \beta_{2}=x_{1}$, and $\beta_{3}=x_{2}$ yielding

$$
x_{n}=\left(x_{2} x_{0}\right)^{G_{n-1}} x_{1}^{G_{n-2}} x_{2}^{G_{n-3}}=x_{0}^{G_{n-1}} x_{1}^{G_{n-2}} x_{2}^{G_{n}} .
$$

This agrees with Lemma 4 in Ref. 9.

Next, we will give a solution of (4) in a slightly simpler form than the one in (5).

Corollary 6 Let the notation be as in Corollary 1. Let $\left\{\mathcal{G}_{n}\right\}$ be the unique sequence satisfying

$\mathcal{G}_{n+\ell}-A_{1} \mathcal{G}_{n+\ell-1}-A_{2} \mathcal{G}_{n+\ell-2}-\cdots-A_{\ell} \mathcal{G}_{n}=0$, with initial values

$$
\mathcal{G}_{0}=\mathcal{G}_{1}=\cdots=\mathcal{G}_{\ell-2}=0, \mathcal{G}_{\ell-1}=1 .
$$

Then the solution of (15) when $n \geqslant \ell$ is

$$
\begin{aligned}
x_{n}=c^{\mathfrak{B}_{n}} & x_{\ell-1}^{A_{1} \mathcal{G}_{n-1}+\cdots+A_{\ell} \mathcal{G}_{n-\ell}} \\
& \cdot x_{\ell-2}^{A_{2} \mathcal{G}_{n-1}+\cdots+A_{\ell} \mathcal{G}_{n-\ell+1}} \\
& \cdots \\
& \cdot x_{1}^{A_{\ell-1} \mathcal{G}_{n-1}+A_{\ell} \mathcal{G}_{n-2}} \\
& \cdot x_{0}^{A_{\ell} \mathcal{G}_{n-1}}
\end{aligned}
$$

where the sequence $\left\{\mathfrak{B}_{n}\right\}$ is as defined in (6).

Proof: In (17) we express all the $\beta_{j}$ 's in terms of the initial values $x_{0}, \ldots, x_{\ell-1}$ neglecting the coefficient term for the time being as this term is more easily computed via (4). This causes no harm due to the uniqueness of the solution. Because of the chosen initial values (22), the sequence $\left\{\mathcal{G}_{n}\right\}$ satisfies no similar recurrence of order lower than $\ell$ and the explicit form (17) together with the system (18) continue to hold with the sequence $\left\{\mathcal{G}_{n}\right\}$ in place of $\left\{G_{n}\right\}$. The choice of the initial values (22) also enables us to easily obtain the $\beta_{j}$ 's from the system (18) successively as

$$
\beta_{j}=\kappa x_{\ell-1}^{A_{j}} x_{\ell-2}^{A_{j+1}} \ldots x_{j-1}^{A_{\ell}} \quad(j=1, \ldots, \ell),
$$

where $\kappa$ denotes the coefficient term independent of the initial values $x_{0}, \ldots, x_{\ell-1}$ and may change from step to step. Substituting these values of $\beta_{j}$ into (17), we get

$$
\begin{aligned}
x_{n}= & \left(\kappa^{\prime}\right)\left(x_{\ell-1}^{A_{1}} x_{\ell-2}^{A_{2}} \cdots x_{0}^{A_{\ell}}\right)^{G_{n-1}} \\
& \cdot\left(x_{\ell-1}^{A_{2}} x_{\ell-2}^{A_{3}} \cdots x_{1}^{A_{\ell}}\right)^{G_{n-2}} \cdots\left(x_{\ell-1}^{A_{\ell}}\right)^{G_{n-\ell}} \\
= & \left(\kappa^{\prime}\right) x_{\ell-1}^{A_{1} G_{n-1}+\cdots+A_{\ell} G_{n-\ell}} x_{\ell-2}^{A_{2} G_{n-1}+\cdots+A_{\ell} G_{n-\ell+1}} \\
& \cdots x_{1}^{A_{\ell-1} G_{n-1}+A_{\ell} G_{n-2}} x_{0}^{A_{\ell} G_{n-1}} .
\end{aligned}
$$

To determine the coefficient term, we use (4) successively starting with

$$
x_{\ell}=c x_{\ell-1}^{A_{1}} x_{\ell-2}^{A_{2}} \cdots x_{1}^{A_{\ell-1}} x_{0}^{A_{\ell}} .
$$

This is the coefficient term in $x_{\ell}=c=c^{\mathfrak{B}_{\ell}}$. Next, from

$$
\begin{aligned}
x_{\ell+1} & =c x_{\ell}^{A_{1}} x_{\ell-1}^{A_{2}} \cdots x_{2}^{A_{\ell-1}} x_{1}^{A_{\ell}} \\
& =c\left(c \cdot \operatorname{term} \text { in } x_{j}\right)^{A_{1}}\left(\text { term in } x_{j}\right) \\
& =c^{1+A_{1}}\left(\operatorname{term} \text { in } x_{j}\right) .
\end{aligned}
$$

This is the coefficient term in

$$
x_{\ell+1}=c^{1+A_{1}}=c^{\mathfrak{B}_{\ell+1}} .
$$

The general case follows at once by induction. 
We indicate how the two rational recursive equations considered by $\mathrm{Li}-\mathrm{Zhu}{ }^{2}$ and two further ones considered by Rhouma ${ }^{9}$ can be easily transformed into the form treated in our main results. We start with the two equations in Ref. 2 ,

$$
\begin{aligned}
& x_{n+3}=\frac{a+x_{n+2} x_{n}}{x_{n+2}+x_{n}} \quad(n=0,1,2, \ldots), \\
& x_{n+3}=\frac{a+x_{n+1} x_{n}}{x_{n+1}+x_{n}} \quad(n=0,1,2, \ldots),
\end{aligned}
$$

where $a \in[0, \infty)$ and the initial values $x_{0}, x_{1}$, and $x_{2}$ are positive. Rewriting (23) and (24), respectively, as

$$
\begin{aligned}
& \left(\frac{x_{n+3}-\sqrt{a}}{x_{n+3}+\sqrt{a}}\right)=\left(\frac{x_{n+2}-\sqrt{a}}{x_{n+2}+\sqrt{a}}\right)\left(\frac{x_{n}-\sqrt{a}}{x_{n}+\sqrt{a}}\right), \\
& \left(\frac{x_{n+3}-\sqrt{a}}{x_{n+3}+\sqrt{a}}\right)=\left(\frac{x_{n+1}-\sqrt{a}}{x_{n+1}+\sqrt{a}}\right)\left(\frac{x_{n}-\sqrt{a}}{x_{n}+\sqrt{a}}\right),
\end{aligned}
$$

and letting $U_{n}:=\frac{x_{n}-\sqrt{a}}{x_{n}+\sqrt{a}}$, the above equations become, respectively,

$$
U_{n+3}=U_{n+2} U_{n}, \quad U_{n+3}=U_{n+1} U_{n},
$$

whose closed-form solutions are deducible from Corollary 1 and from which their global asymptotic stability can be analysed.

Next, we consider two more rational recursive equations taken from sections 4 and 5.2 of Ref. 9 starting with

$$
y_{n+2}=\frac{y_{n-k+1} y_{n-j+1}+a}{y_{n-k+1}+y_{n-j+1}},
$$

where $a>0$, and $k, j \in \mathbb{N}, k>j$. Equation (25) is equivalent to

$$
\left(\frac{y_{n+2}-\sqrt{a}}{y_{n+2}+\sqrt{a}}\right)=\left(\frac{y_{n-k+1}-\sqrt{a}}{y_{n-k+1}+\sqrt{a}}\right)\left(\frac{y_{n-j+1}-\sqrt{a}}{y_{n-j+1}+\sqrt{a}}\right) .
$$

Letting $U_{n}:=\left(y_{n}-\sqrt{a}\right) /\left(y_{n}+\sqrt{a}\right)$, the above equation becomes $U_{n+2}=U_{n-k+1} U_{n-j+1}$, or $U_{n+k+1}=U_{n} U_{n+k-j}$, which is of the desired form.

Now for our final application, consider the rational recursive equation

$$
x_{n+k+1}=a \frac{x_{n+k}-x_{n}}{x_{n}+a}(n \geqslant 0),
$$

where $k \in \mathbb{N}, k>1, a \in \mathbb{R}$ and $x_{0}, \ldots, x_{k}$ are given initial values none of which is equal to $-a$. Via the substitution $x_{n}=a\left(y_{n}-1\right)$, (26) is equivalent to

$$
a\left(y_{n+k+1}-1\right)=a \frac{a\left(y_{n+k}-1\right)-a\left(y_{n}-1\right)}{a\left(y_{n}-1\right)+a},
$$

or $y_{n+k+1}=y_{n+k} y_{n}^{-1}$, which is of the desired form.

\section{CONTINUED FRACTIONS}

The case $\ell=2$ of Theorem 1 is closely connected with continued fractions and we will show that the exponents in the solution (11) can be read off from the numerators and denominators of convergents of a specific continued fraction. We give the case $\ell=2$ of Corollary 1 with the $\beta_{j}$ 's explicitly computed.

Corollary 7 Let $c, h_{1}, h_{2}$ be three non-zero complex constants. Let $\left\{G_{n}\right\}_{n \geqslant 0}$ be the unique sequence satisfying

$$
G_{n}-h_{1} G_{n-1}-h_{2} G_{n-2}=0 \quad(n \geqslant 2),
$$

with given initial values $G_{0}, G_{1}$ chosen so that $\mathcal{B}:=$ $G_{1} G_{-1}-G_{0}^{2} \neq 0$, where $G_{-1}:=\frac{G_{1}-h_{1} G_{0}}{h_{2}}$. If $\left\{x_{n}\right\}_{n \geqslant 0}$ is a sequence of complex numbers satisfying the recursive equation

$$
x_{n}=c x_{n-1}^{h_{1}} x_{n-2}^{h_{2}} \quad(n \geqslant 2),
$$

with given initial values $x_{0}, x_{1}$ chosen so that all remaining $x_{n}$ are uniquely well-defined, then the solution of (28) is given by

$$
\begin{aligned}
x_{n}=P_{c}(n) & \left(\frac{x_{0}}{P_{c}(0)}\right)^{\frac{G_{1} G_{n-1}-G_{0} G_{n}}{\mathcal{B}}} \\
& \cdot\left(\frac{x_{1}}{P_{c}(1)}\right)^{\frac{G_{-1} G_{n}-G_{0} G_{n-1}}{\mathcal{B}}} \quad(n \geqslant 2),
\end{aligned}
$$

where $P_{c}(n)$ is a particular solution of (28).

A particular case of Corollary 7 where $c=1, G_{0}=0$, $G_{1}=1$ gives rise to the following corollary.

Corollary 8 Let $h_{1}, h_{2}$ be two non-zero complex constants. Let $\left\{G_{n}\right\}_{n \geqslant 0}$ be the unique sequence satisfying

$$
G_{n}-h_{1} G_{n-1}-h_{2} G_{n-2}=0 \quad(n \geqslant 2),
$$

with given initial values $G_{0}=0, G_{1}=1$. If $\left\{x_{n}\right\}_{n \geqslant 0}$ is a sequence of complex numbers satisfying the recursive equation

$$
x_{n}=x_{n-1}^{h_{1}} x_{n-2}^{h_{2}} \quad(n \geqslant 2),
$$

with given initial values $x_{0}, x_{1}$ chosen so that all remaining $x_{n}$ are uniquely well-defined, then the solution of (29) is given by

$$
x_{n}=x_{0}^{h_{2} G_{n-1}} x_{1}^{G_{n}} \quad(n \geqslant 2) .
$$


Next, let us review some relevant facts about continued fractions ${ }^{14,15}$. Define the sequence $\left\{S_{n}\right\}_{n} \geqslant 1$ by

$$
\begin{gathered}
S_{n}=b_{0}+\frac{a_{1}}{b_{1}+\frac{a_{2}}{b_{2}+\frac{a_{3}}{b_{3}+\frac{a_{n}}{b_{n}}}}} \\
\quad:=b_{0}+\left[a_{1} / b_{1}, a_{2} / b_{2}, \ldots, a_{n} / b_{n}\right] \quad(n \geqslant 1) .
\end{gathered}
$$

If the sequence $\left\{S_{n}\right\}_{n \geqslant 1}$ converges with respect to some appropriate topology, we write

$$
\begin{aligned}
S_{\infty}: & =\lim _{n \rightarrow \infty} S_{n}=b_{0}+\frac{a_{1}}{b_{1}+\frac{a_{2}}{b_{2}+\frac{a_{3}}{b_{3}+} \cdot}} \\
& =b_{0}+\left[a_{1} / b_{1}, a_{2} / b_{2}, \ldots\right]
\end{aligned}
$$

and call it a (non-regular) continued fraction of the element it represents. $S_{n}$ is called the $n$th convergent of the continued fraction (30). If

$$
a_{1}=a_{2}=\cdots=1 \text { and } b_{i} \in \mathbb{N} \quad(i \geqslant 1),
$$

then (30) is customarily denoted by $\left[b_{1}, b_{2}, b_{3}, \ldots\right]$. Let

$$
p_{-1}=1, p_{0}=b_{0}, q_{-1}=0, q_{0}=1,
$$

and define $p_{n}, q_{n}$ as the numerator and denominator in the expression

$$
\frac{p_{n}}{q_{n}}:=S_{n} \quad(n \geqslant 1)
$$

It is well-known (see e.g., Chapter 2 of Ref. 14 or Chapter 1 of Ref. 15) that the sequences $\left\{p_{n}\right\}_{n \geqslant 1}$ and $\left\{q_{n}\right\}_{n \geqslant 1}$ satisfy the same second order linear recurrence relation (but with different initial conditions); for $n \geqslant 1$,

$$
\begin{gathered}
p_{n}=b_{n} p_{n-1}+a_{n} p_{n-2}, \\
q_{n}=b_{n} q_{n-1}+a_{n} q_{n-2} .
\end{gathered}
$$

Combining the two concepts of continued fractions and recursive equations in the case $\ell=2$ of Theorem 1, we obtain the following theorem.

Theorem 2 Let $h_{1}, h_{2}$ be two functions from $\mathbb{N}_{0}$ to $\mathbb{C}$ with $h_{1}(n) h_{2}(n) \neq 0$ for all $n$. Let

$$
p_{0}=h_{1}(0), p_{1}=h_{1}(0) h_{1}(1)+h_{2}(1) \text {, }
$$

$q_{0}=1, q_{1}=h_{1}(1)$, and let $p_{n}$ and $q_{n}$ be the numerator and denominator of the finite continued fraction

$$
h_{1}(0)+\left[h_{2}(1) / h_{1}(1), \ldots, h_{2}(n) / h_{1}(n)\right] .
$$

If $\left\{x_{n}\right\}_{n \geqslant 0}$ is a sequence of complex numbers satisfying the recursive equation

$$
x_{n}=x_{n-1}^{h_{1}(n)} x_{n-2}^{h_{2}(n)} \quad(n \geqslant 2),
$$

with given initial values $x_{0}, x_{1}$ chosen so that all remaining $x_{n}$ are uniquely well-defined, then the solution of (36) is given by

$$
x_{n}=x_{0}^{G_{n}^{(1)}} x_{1}^{G_{n}^{(2)}} \quad(n \geqslant 2),
$$

where

$$
\begin{aligned}
G_{n}^{(1)} & =\frac{\left\{h_{2}(1)+h_{1}(0) h_{1}(1)\right\} q_{n}-h_{1}(1) p_{n}}{h_{2}(1)}, \\
G_{n}^{(2)} & =\frac{p_{n}-h_{1}(0) q_{n}}{h_{2}(1)} .
\end{aligned}
$$

Proof: We obtain the solution (37) by mathematical induction.

Remark 3 In the same manner as in Remarks 1 and 2 , the following formal manipulation could be used to get the form of solutions. Taking the logarithm of the equation (36) turns it into the linear difference equation

$\log x_{n}=h_{1}(n) \log x_{n-1}+h_{2}(n) \log x_{n-2} \quad(n \geqslant 2)$.

Let $\left\{\log x_{n}^{(p)}\right\}_{n \geqslant 0}$ and $\left\{\log x_{n}^{(q)}\right\}_{n \geqslant 0}$ be two sequences satisfying the same recurrence (38) but with initial conditions

$$
\begin{aligned}
h_{1}(0) & =\log x_{0}^{(p)}, h_{1}(0) h_{1}(1)+h_{2}(1)=\log x_{1}^{(p)}, \\
1 & =\log x_{0}^{(q)}, h_{1}(1)=\log x_{1}^{(q)} .
\end{aligned}
$$

Thus $\log x_{n}^{(p)}$ and $\log x_{n}^{(q)}$ are simply the numerator $p_{n}$, and the denominator $q_{n}$, of the finite continued fraction (35). Theorem 1 gives us a general solution of (36) as

$$
x_{n}=x_{0}^{G_{n}^{(1)}} x_{1}^{G_{n}^{(1)}} .
$$

Taking the principal logarithm of the equation (39), we get

$$
\begin{gathered}
p_{n}:=\log x_{n}^{(p)}=G_{n}^{(1)} \log x_{0}^{(p)}+G_{n}^{(2)} \log x_{1}^{(p)} \\
=h_{1}(0) G_{n}^{(1)}+\left(h_{1}(0) h_{1}(1)+h_{2}(1)\right) G_{n}^{(2)},
\end{gathered}
$$


and

$$
\begin{aligned}
& q_{n}:=\log x_{n}^{(q)}=G_{n}^{(1)} \log x_{0}^{(q)}+G_{n}^{(2)} \log x_{1}^{(q)} \\
& =G_{n}^{(1)}+h_{1}(1) G_{n}^{(2)} .
\end{aligned}
$$

Solving (40) and (41), the desired result follows.

If the coefficients functions $h_{i}(n)$ in Theorem 2 are constants, using the same proof as in Theorem 2 as well as the result of Corollary 8 , we have the following theorem.

Theorem 3 Let $h_{1}, h_{2}$ be two nonzero complex numbers. If $\left\{x_{n}\right\}_{n \geqslant 0}$ satisfies the recursive equation

$$
x_{n}=x_{n-1}^{h_{1}} x_{n-2}^{h_{2}} \quad(n \geqslant 2),
$$

with given initial values $x_{0}, x_{1}$ chosen so that all remaining $x_{n}$ are uniquely well-defined, then the solution of (42) is given by

$x_{n}=\left(x_{0}^{-h_{1} p_{n}+\left(h_{1}^{2}+h_{2}\right) q_{n}} x_{1}^{p_{n}-h_{1} q_{n}}\right)^{1 / h_{2}} \quad(n \geqslant 2)$,

where $p_{0}=h_{1}, p_{1}=h_{1}^{2}+h_{2}, q_{0}=1, q_{1}=h_{1}$, and $p_{n}$ and $q_{n}$ are the numerator and denominator of the finite continued fraction

$$
h_{1}+\left[h_{2} / h_{1}, \ldots, h_{2} / h_{1}\right] \quad(n \geqslant 2)
$$

consisting of the same fraction $h_{2} / h_{1}$ repeated $n$ times.

We now apply the results of Theorems 2 and 3 to derive solutions of a number of recursive equations in terms of numerators and denominators of specific continued fractions.

Example 4 If $h_{1}(n)=1=h_{2}(n)$, then the solution of the rational recursive equation

$$
x_{n}=x_{n-1} x_{n-2} \quad(n \geqslant 2)
$$

with given initial values $x_{0}, x_{1}$ chosen so that all remaining $x_{n}$ are uniquely well-defined, is given by

$$
x_{n}=\left(x_{0}^{-p_{n}+2 q_{n}} x_{1}^{p_{n}-q_{n}}\right) \quad(n \geqslant 2),
$$

where $p_{0}=1, p_{1}=2, q_{0}=1, q_{1}=1$, and $p_{n}$ and $q_{n}(n \geqslant 2)$ are the numerator and denominator of the finite continued fraction

$$
1+\underbrace{[1 / 1,1 / 1, \ldots, 1 / 1]}_{n \text { terms }} .
$$

In this case, sequences satisfying (27) include the sequence of Fibonacci numbers and the sequence of Lucas numbers, which contains the work of Rhouma ${ }^{9}$.
Example 5 If $h_{1}(n)=x, h_{2}(n)=1$, then the solution of the recursive equation

$$
x_{n}=x_{n-1}^{x} x_{n-2} \quad(n \geqslant 2)
$$

with given initial values $x_{0}, x_{1}$ chosen so that all remaining $x_{n}$ are uniquely well-defined, is given by

$$
x_{n}=x_{0}^{-x p_{n}+\left(x^{2}+1\right) q_{n}} x_{1}^{p_{n}-x q_{n}} \quad(n \geqslant 2),
$$

where $p_{0}=x, p_{1}=x^{2}+1, q_{0}=1, q_{1}=x$, and $p_{n}$ and $q_{n}(n \geqslant 2)$ are the numerator and denominator of the finite continued fraction

$$
x+\underbrace{[1 / x, 1 / x, \ldots, 1 / x]}_{n \text { terms }} .
$$

In this case, sequences satisfying (27) include the sequence of Fibonacci polynomials and the sequence of Lucas polynomials.

Example 6 If $h_{1}(n)=2 x, h_{2}(n)=-1$, then the solution of the recursive equation

$$
x_{n}=x_{n-1}^{2 x} x_{n-2}^{-1} \quad(n \geqslant 2),
$$

with given initial values $x_{0}, x_{1}$ chosen so that all remaining $x_{n}$ are uniquely well-defined, is given by

$$
x_{n}=x_{0}^{2 x p_{n}-\left(4 x^{2}-1\right) q_{n}} x_{1}^{-p_{n}+2 x q_{n}} \quad(n \geqslant 2),
$$

where $p_{0}=2 x, p_{1}=4 x^{2}-1, q_{0}=1, q_{1}=$ $2 x$, and $p_{n}$ and $q_{n},(n \geqslant 2)$ are the numerator and denominator of the finite continued fraction

$$
2 x+\underbrace{[-1 / 2 x,-1 / 2 x, \ldots,-1 / 2 x]}_{n \text { terms }} .
$$

In this case, sequences satisfying (27) include the sequence of Chebyshev polynomials.

Example 7 If $h_{1}(n)=2 n+3-x, h_{2}(n)=-(n+$ $1)^{2}$, then the solution of the recursive equation

$$
x_{n}=x_{n-1}^{2 n+3-x} x_{n-2}^{-(n+1)^{2}} \quad(n \geqslant 2),
$$

with given initial values $x_{0}, x_{1}$ chosen so that all remaining $x_{n}$ are uniquely well-defined, is given by

$$
x_{n}=x_{0}^{\frac{1}{4}\left((5-x) p_{n}-\left(x^{2}-8 x+11\right) q_{n}\right)} x_{1}^{\frac{1}{4}\left((3-x) q_{n}-p_{n}\right)},
$$

where $p_{0}=3-x, p_{1}=11-8 x+x^{2}, q_{0}=1$, $q_{1}=5-x$, and $p_{n}$ and $q_{n}(n \geqslant 2)$ are the numerator and denominator of the finite continued fraction

$3-x+[-4 /(5-x),-9 /(7-x), \ldots$

$$
\left.\ldots,-(n+1)^{2} /(2 n+3-x)\right] .
$$

In this case, sequences satisfying (8) include the sequence of shifted Laguerre polynomials. 
Example 8 If $h_{1}(n)=2 x, h_{2}(n)=-2(n+1)$, then the solution of the recursive equation

$$
x_{n}=x_{n-1}^{2 x} x_{n-2}^{-2(n+1)} \quad(n \geqslant 2),
$$

with given initial values $x_{0}, x_{1}$ chosen so that all remaining $x_{n}$ are uniquely well-defined, is given by

$$
x_{n}=x_{0}^{\frac{1}{2}\left(x p_{n}+\left(2-2 x^{2}\right) q_{n}\right)} x_{1}^{\frac{1}{4}\left(2 x q_{n}-p_{n}\right)} \quad(n \geqslant 2),
$$

where $p_{0}=2 x, p_{1}=4 x^{2}-4, q_{0}=1, q_{1}=2 x$, and $p_{n}$ and $q_{n}(n \geqslant 2)$ are the numerator and denominator of the finite continued fraction

$$
2 x+[-4 / 2 x,-6 / 2 x, \ldots,-2(n+1) / 2 x] .
$$

In this case, sequences satisfying (8) include the sequence of shifted Hermite polynomials.

Example 9 If $h_{1}(n)=\frac{2 n+3}{n+2} x, h_{2}(n)=\frac{-(n+1)}{n+2}$, then the solution of the recursive equation

$$
x_{n}=x_{n-1}^{(2 n+3) x /(n+2)} x_{n-2}^{-(n+1) /(n+2)} \quad(n \geqslant 2),
$$

with given initial values $x_{0}, x_{1}$ chosen so that all remaining $x_{n}$ are uniquely well-defined, is given by

$x_{n}=x_{0}^{\frac{1}{4}\left(10 x p_{n}-\left(15 x^{2}-4\right) q_{n}\right)} x_{1}^{\frac{1}{4}\left(9 x q_{n}-6 p_{n}\right)} \quad(n \geqslant 2)$,

where $p_{0}=\frac{3}{2} x, p_{1}=\frac{5}{2} x^{2}-\frac{2}{3}, q_{0}=1, q_{1}=\frac{5}{3} x$, and $p_{n}$ and $q_{n}(n \geqslant 2)$ are the numerator and denominator of the finite continued fraction

$\frac{3 x}{2}+\left[\frac{-2}{3} / \frac{5 x}{3}, \frac{-3}{4} / \frac{7 x}{4}, \ldots, \frac{-(n+1)}{n+2} / \frac{(2 n+3) x}{n+2}\right]$.

In this case, sequences satisfying (8) include the sequence of shifted Legendre polynomials.

Acknowledgements: This study was supported by the Thailand Research Fund (RTA 5180005), the Centre of Excellence in Mathematics, the Commission on Higher Education, and the KU Centre for Advanced Studies, Thailand.

\section{REFERENCES}

1. Kocic VL, Ladas G (1993) Global Behaviour of Nonlinear Difference Equation of Higher Order with Applications, Kluwer Academic Press, Dordrecht.

2. Li X, Zhu D (2004) Two rational recursive sequences. Comput Math Appl 47, 1487-94.

3. Aloqeili M (2009) Global stability of a rational symmetric difference equation. Appl Math Comput 215, 950-3.

4. Aprahamian M, Souroujon D, Tersian S (2010) Decreasing and fast solutions for a second-order difference equation related to Fisher-Kolmogorov's equation. J Math Anal Appl 363, 97-110.
5. Memarbashi R (2008) Sufficient conditions for the exponential stability of nonautonomous difference equations. Appl Math Lett 21, 232-5.

6. Wang C, Wang S, Li L, Shi Q (2009) Asymptotic behavior of equilibrium point for a class of nonlinear difference equation, Advances in Difference Equations, Volume 2009, Article ID 214309. 8 pages.

7. Wang C, Wang S, Wang W (2011) Global asymptotic stability of equilibrium point for a family of rational difference equations. Appl Math Lett 24, 714-8.

8. Wang C, Wang S, Wang Z, Gong H, Wang R (2010) Asymptotic stability for a class of nonlinear difference equation, Discrete Dynamics in Natural and Society, Volume 2010, Article ID 791610, 10 pages.

9. Rhouma MBH (2005) The Fibonacci sequence modulo $\pi$, chaos and some rational recursive equations. $J$ Math Anal Appl 310, 506-17.

10. Hengkrawit C, Laohakosol V, Udomkavanich P (2010) Rational recursive equations characterizing cotangenttangent and hyperbolic cotangent-tangent functions. Bull Malays Math Sci Soc 33, 421-8.

11. Gelfond A (1971) Calculus of Finite Differences, Hindustan Publishing Cooperation, Delhi.

12. Elaydi S (2005) An Introduction to Difference Equations, 3rd edn, Springer, New York.

13. van der Poorten AJ (1976) Some determinants that should be better known. J Austral Math Soc Ser A 21, 278-88.

14. Jones WB, Thron WJ (1980) Continued Fractions: Analytic Theory and Applications, Encyclopedia of Mathematics and its Applications Vol.11, AddisonWesley, Reading.

15. Lorentzen L, Waadeland H (1992) Continued Fractions with Applications, North-Holland, Amsterdam. 